so fand ich doch, dafs derselbe von Kindern nicht immer gut vertragen wurde und entweder Stuhlverstopfung oder übelriechende, schwarz tingirte Stühle verursachte; of wollten die Kinder den Eichelkaffee wegen seines unangenehmen, säuerlichen, bittern Geschmackes nicht trinken, indem die zugegossede Milch häufig gerann. Ich lasse daher, um dem Eichelkaffee die Gallussäure zu benchmen, ihn ouf folgende Weise bereiten: Von den besten grofsen Eicheln der Quercus pedunculata lasse ich roh in vier Stücke zerschmeiden, diese zweimal nit siedendem Wasser anbrühen und bis zum Erlalten stehen, sodann werden die Eicheln im Bacliofen gedörrt und nun erst ihre doppelten Schalen abgeschält und gerōstet. Durch dieses Verfahı'en wird der Eichellıffee ein angenehmes, leicht vertı̈̈gliches, starkes Getränk, indem die Eicheln dadurch ihre überschüssige Gallussäure verlieren.

(Würtemb. Medicin. Correspondenzblatt Bd. VI. Nro. 34.)

\title{
Resultate von Versuchen und Beobachtungen, die mit Kreosot bei Menschen und Thieren angestellt wurden; von Professor Corneliani in Pavia.
}

Corneliani machte das fireosot zum Gegenstand seiner Untersuchungen, sowobl in seiner Klinil, als auch bei mehreren Thieren, ohne die Anpreisungen dieses Mittels gegen vorschiedene Affectionel, von Reichenbach, Buchner, Having, unmittelbar anzunehmen.

Er machte davon sowohl innerlich, als auch dorch dio ondermische Methode, so wie durch Eiuspritzung in die Venen Gebrauch, indem er von den kleinsten Dosen bis zu den höchsten stieg. Aus seizen Versuchen gehen folgende Resultate hervor: 
1) Das If:cosot in starker Dosis innerlich genommen, kann ant der stelle den Tod bervorbringen, ohne dafs Au. topsie, wenn sie sogleich nach dem 'lode geschieht, irgend eine organiscbe Verleizung erkennen lüfst.

9) Wird reines oder nur wenig verdiinntes Kreosot auf cinen starken blosgelegten Nerven, welcher es auch sey, gebracht, oder wird es selbst in sehr kleiner Dosis in eine Ader eingespritzt, so erfolgt unmittelbar der Tod.

3) Wenn die Gabe nicht grois genug ist, uro den Tod herrorzubringen, so veranlafst es eine Eistarrung in dem Muskel - und Nervensystem, Iäbmung der Extremitäten, des Herzens, des Zwergfells und der Sinnesorgane, so dals man dem Hreosot die Wirkung der sehwächenden Narcotica zuschreiben liann, unter welche es anch eingereiht werden mufs.

4) Obschon man bis jetzt noch kein Antidotum gegen die giftigen Wirkungen dieser Substanz bennt, so scheineo doch Stimulantia indicirt zu seyn, wenn noch heine Wirkang auf den Magen vorhanden ist; denn in diesem Falle würden sie, wie bei Narcoticis, als wie Kirschlorbeerwassor, die tődtlichen Wirkungen vermebren.

5) Das Líreosot übe auf dic Magen - und Darm-Schleim. hant eine mehr oder weniger slarke mecbanisch-chemiscbe Einwirkung aus, dit zu den verschiedenen organischen Vorletzangen, welche unan auf dem Körper findet, and zn chro. nischen Darmentzündungen Veranlassung gibt, welchen die Personen, die lange Zeit Kreosot gebranchten, unterworfen sind.

6) Um dieser durch innerlichen Gebrauch des Hreosots Lerrorgebrachten mechanischehemuschen Wirkung entgegenzuwirken, mufs man von öligen und schleimigen Getränken Gebrauch machen. Essig, welcher ein treffliches Anflösungsmittel dieser Substanz ist, würde nur die gefährlichen Wir- 
kungen vermehren, indem er sie mit den nervösen Warzen des Magens mehr in Berührung bringt.

7) Im Allgemeinen können die Kranken eine Gabe über 2 Tropfen, 4- bis 6mal innerhalb 24 Stunden wiederholt, nicht crtragen.

8) Das Hreosot hann innerlich genommen bei der zuckerigen Hernruhr, Blutspeien, chronischen Catarrhen, Diarrboe, Herzulopfen, und vielleicht selbst bei Tetanus mit Nutzen angewendet werden.

9) Eine sonderbare Wirkung des innerlich genommenen Kreosots scheint zuerst auf die Harnwege stattzufiaden, denn ein Thier, mit welchem man experimentirte, urivirte sogleich, nachdem es genommen hatte. Corneliani schreibt diefs besonders einer Lähmung des Blasenhaises zu.

10) Die äurserliche Anwendung des Kreosots bei chronischen Hautkranthoiten und vorzügiich Flechten, Irötze and chronischer Poriasis kann von Erfolg seyn, weil die Wirknng entweder austrocluend, antiphlogistisch oder Insectentōdtend (besonders bei Krätze) ist.

11) Das Hreosot ist sebr wirksam bei Wunden und einfachen Geschwüren, welche es reinigt und leicht vernarbt.

12) Wenn das Kreosot äufserlich nicht auf einer grofsen Oberfläche oder auf wichtigen Nervenverzweigungen angewendet wird, so erzeugt es heine Veränderung der Organe, selbst derjenigen, auf welche es mehr speciell wirkt, uls das Rückenmark, Gebirn und Nieren. Corneliani hat jedoch beabachtet, dafs bei Schwindsuchten die Einathmung des Kreosots eine Fistarrung der intellectuellen Functionen und der Muskelbewegungen stattfinden könne.

13) Wenn die Anwendung des Kreosots die nervösen Blutungen, am wenigsten die aus einer starken Vene, wie z. B. die Schenkelvene eines Schafs, nicht stillt, so ist es doch wirksam bei Blutung einer geringeren Arterie. 
Anmerkung der Redaction des Journ. de Chim. medic.

Mehrere Beobachtungen des italienischen Professors stimmen init denen der Herren Ollivier, Coster, Yvan, Berthelot, Iuc und Martin Solon überein. Letzterer wendete das Kreosot mit Erfolg zur Conservation anatomischer Präparate an.

Anch ist es bekannt, dafs das Kreosot eine besondere Wirkung auf Eiweifs zeigt, welches schnell coagulirt wird, chen so eine erregende Wirkung anf die thicrische Oetsono. mie, was zu erkepnen gibt, dafs es nur in sehr verdünntem Zustande angewendet werden darf.

(Journ, de Chimie medic. Ferricr 1836.)

\section{Behandlung von Brandverletzungen durch Phagadänisches Wasser und Rhabarbar- pulver.}

Hintze erzöhlt acht Fälle von Brandwunden, die entweder durch tochendheifse Flüssigkeiten, durch schmelzendes Metall oder durch brennende Substanzen hervorgebracht worden waren, und in allen diesen Fällen war seine Methode von Erfolg.

Er schnitt mit der Scheere die durch die Blasenbildang aufgetriebene Epidermis weg, befeuchtete die Wunde mit phagadönischem Wasser vermittelst eine. Feder, und streute dann Rhabarbar darauf. Er nahm einen Gran Sublimat auf' eine Unze Lïsungsmittel bei frischen Brandwunden, bei chronischen Geschwüren stieg ev mit der Dosis viel hüber.

(Journ. de connaiss. medico chirurg. Fevrier 1936.) 